\title{
Effect of cement fineness and polycarboxylate dosage on the rheological and mechanical behavior of a mortar
}

\author{
Didouche Zahia ${ }^{1}$, Ezziane Karim ${ }^{1}$. \\ ${ }^{1}$ Hassiba Benbouali University, Civil Engineering Department, Chlef, Algeria.
}

\begin{abstract}
The use of certain organic additives in the production of mortar and concrete influences the workability and the hydration kinetic of mortar. This results in a modification of some properties, namely rheological behavior and mechanical strength. The objective of this work is to evaluate the rheological and mechanical behavior of a mortar by varying the fineness of the cement and using the superplasticizer Polycarboxylate.
\end{abstract}

\section{INTRODUCTION}

A l'état frais, la présence des additions minérales modifie la structure du squelette granulaire et les frictions entre les composants solides dans la phase liquide. Certaines additions peuvent réagir chimiquement en milieu cimentaires pour former de nouveaux produits hydratés qui présentent un caractère liant supplémentaire.

La petite taille des particules du filler calcaire comble les vides interstitiels et augmente la densité de la matière. Par conséquent, l'eau attrapée dans les pores granulaires est libérée ce qui augmente la compacité de la pâte, améliore l'ouvrabilité et diminue la demande en eau [1, 2, $3]$. Les dosages élevés en filler calcaire augmentent la viscosité de la pâte [1], Le seuil de cisaillement augmente avec la quantité d'addition ultrafine incorporée tandis que la viscosité de la pâte varie avec la nature et la quantité d'addition [4]. D'autre part une augmentation de la finesse offre une ouvrabilité satisfaisante car le clincker adhère mieux au calcaire et la demande en eau diminue par rapport à celle du ciment portland [5]. Aussi la composition chimique d'un ciment est le facteur le plus important qui influence l'hydratation [6]. L'utilisation des fillers augmente le taux d'hydratation [7]. Le calcaire favorise la cristallisation des monocarbonates plus que les monosulfates et stabilise indirectement l'ettringite [8]. Il intervient lors de l'hydratation des $\mathrm{C}_{3} \mathrm{~A}$ pour former carboaluminate et retarde la transformation d'ettringite en monosulfoaluminate, il constitue des sites de nucléation pour les cristaux de l'hydroxyde de calcium au jeune âge de l'hydratation $[9,3]$, cette addition améliore la réactivité $\mathrm{du}$ clincker et exploite son potentiel hydraulique [10], mais le flux de chaleur total après $72 \mathrm{~h}$ d'hydratation est similaire à celui du ciment portland due à l'effet de dilution [8].
En outre un ciment fin possède une grande surface spécifique avec une large surface de contact avec l'eau ce qui augmente le taux d'hydratation, ainsi les produits hydratés seront de faible épaisseur autour des particules de ciment anhydre et causent une élévation du degré d'hydratation [6]. D'après une étude de recherche, un ciment contenant les particules fines du filler calcaire montre un temps de prise court [9], mais Voglis et al. [4] ont déclaré que l'addition calcaire accélère le début de prise et retarde la fin de prise, par conséquent le temps de prise est prolongé par rapport à celui du ciment portland. Pour d'autres [11], le début et la fin de prise varient en fonction du dosage en calcaire. Une addition de $5 \%$ de calcaire augmente la plasticité de la pâte et prolonge le temps de prise, mais pour un dosage en calcaire supérieur à $20 \%$ le début et la fin de prise diminuent.

L'ajout des superplastifiants au mortier améliore ses propriétés dû à la baisse du rapport $\mathrm{E} / \mathrm{C}$ nécessaire. Les polymères affectent l'interface entre la surface des particules et la solution interstitielle et influencent les propriétés physiques telles que la viscosité et la limite d'élasticité de la pâte $[12,13]$. Les superplastifiants à base de naphtalène et de mélamine augmentent les propriétés rhéologiques des pâtes de ciment à court et à long termes à cet effet la réduction de la viscosité plastique et le seuil de cisaillement dépendent de la composition du ciment et du temps d'introduction du superplastifiant [14]. Ainsi le type de superplastifiant à une grande influence sur le comportement rhéologique des mortiers de ciment à l'état frais et que les superplastifiants à base de polycarboxylate étaient plus efficaces que les superplastifiants à base de naphtalène [15,13]. Pour Heikal et al. [16] le superplastifiant à base de polycarboxylate adsorbé sur les surfaces des particules du ciment crée une charge 
négative sur les grains du ciment causant une répulsion électrostatique ce qui augmente les distances entre les particules et améliore la fluidité de la pâte .Si le mortier est considéré comme un ensemble de couches granulaires parallèles soumises à une contrainte tangentielle.

Si une des couches du mortier se déplace par rapport à celle qui lui est sous-jacente en raison du frottement permanent sur les grains de la seconde couche, le mouvement est transmis partiellement à cette dernière en même temps que la vitesse de déplacement de la première couche diminue. Cet effet de retard, provoqué par la friction interne des grains de la couche sous-jacente sur celle de la couche supérieure, est appelé viscosité

$\mu$ [Pa.s]. Donc, la viscosité peut être définie comme la résistance au glissement d'un système soumis à une contrainte tangentielle. Elle est caractérisée par un coefficient de viscosité qui joue un rôle essentiel dans la rhéologie des fluides. Pour certains fluides, sa connaissance suffit à caractériser de façon précise son comportement rhéologique [17].

\section{Etude expérimentale}

\subsection{Matériaux utilisés}

Le ciment utilisé dans ce travail est un ciment portland composé (CEM II/42.5) contenant $12 \%$ de calcaire broyé à des temps variés pour donner quatre types de ciment (Tableau1). Ces ciments sont utilisés pour confectionner des mortiers à base d'un sable normalisé. Un superplastifiant à base de polycarboxylate noté (PC) fourni par l'entreprise GRANITEX et commercialisé sous le nom de MEDAFLOW 30 a été utilisé. Ce superplastifiant a été dilué dans l'eau de gâchage et incorporé dans la composition du mortier avec différents dosages.

Tableau I. Finesse des ciments utilisés.

\begin{tabular}{|l|l|l|l|l|}
\hline Ciment & C1 & C2 & C3 & C4 \\
\hline $\begin{array}{l}\text { Finesse } \\
\left(\mathrm{cm}^{2} / \mathrm{g}\right)\end{array}$ & 3026 & 3298 & 3668 & 4000 \\
\hline
\end{tabular}

\subsection{Confection des mortiers}

Les mélanges destinés à mesurer les paramètres rhéologiques et la résistance mécanique sont confectionnés sous une température constante de $20^{\circ} \mathrm{C}$ avec différentes teneurs en superplastifiant. En soustrayant la quantité d'eau contenue dans le superplastifiant de la quantité d'eau de gâchage pour chaque dosage utilisé (Tableau 2).

Tableau II . Compositions des échantillons de mortier.

\section{Présentation et discussion des résultats}

\subsection{Rhéologie}

La "figure 1" montre la variation du seuil de cisaillement $\tau_{0}$ des mortiers en fonction du dosage en Polycarboxylate et de la finesse du ciment.

La comparaison du comportement rhéologique des différents mortiers permet de constater en premier lieu que le mortier adjuvanté présente des taux de cisaillement significativement plus faibles que les mortiers nonadjuvantés. Ceci est inhérent avec le rôle dispersant de l'adjuvant et la diminution des forces de frottement entre les grains lors du cisaillement qu'il engendre. D'autre part, les mortiers à base de ciment à finesse élevée ont un seuil de cisaillement plus important que les mortiers confectionnés avec des ciments à faible finesse, ceci peut être expliqué par l'augmentation de la finesse qui comble les vides dans la pâte cimentaire et augmente les forces de frottement entre les grains ce qui conduit à une augmentation du seuil de cisaillement dans la pâte.

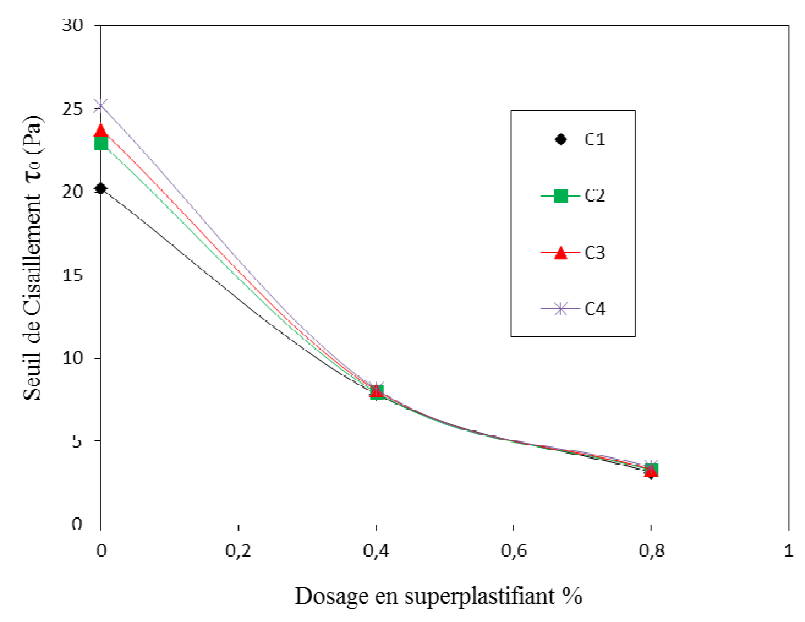

Fig.1. Effet du dosage en Polycarboxylate et de la finesse du ciment sur le seuil de cisaillement des mortiers.

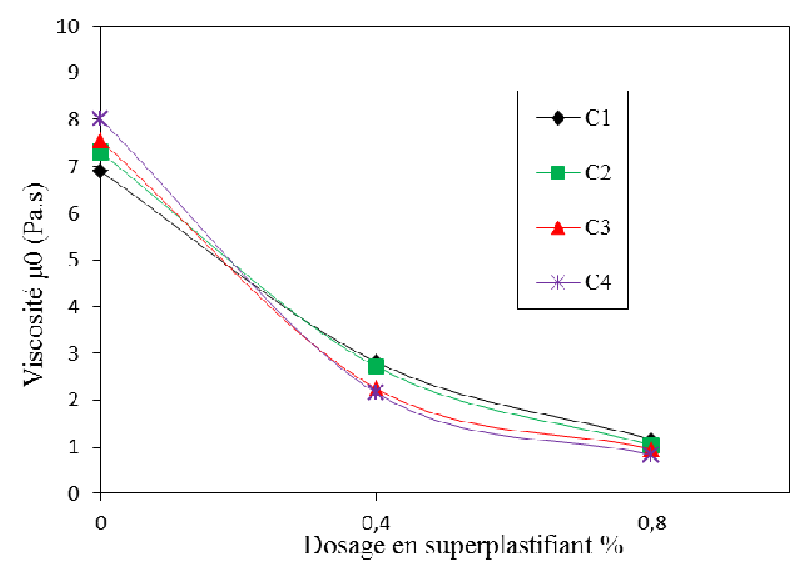

Fig.2. Effet de la finesse et du dosage en plolycarboxylate sur la viscosité des différents mortiers.

\begin{tabular}{|l|c|c|c|c|c|}
\hline L'essai & E/C & $\begin{array}{c}\text { Ciment } \\
(\mathrm{g})\end{array}$ & $\begin{array}{c}\text { Sable } \\
(\mathrm{g})\end{array}$ & $\begin{array}{c}\text { Eau } \\
(\mathrm{g})\end{array}$ & PC (\%) \\
\hline Rhéologie & 0.5 & 630 & 1890 & 315 & $0,0.4,0.8$ \\
\hline $\begin{array}{l}\text { Résistance } \\
\text { mécanique }\end{array}$ & 0.5 & 450 & 1350 & 225 & $0,0.4,0.8$ \\
\hline
\end{tabular}


Les résultats portés sur la "figure 2" montre une chute considérable de la valeur de viscosité lorsque le dosage en Polycarboxylate augmente, cette chute est autant plus significative pour un dosage supérieur à $0.4 \% \mathrm{PC}$. Ce résultat s'aligne aux travaux de Puertas et al. [12] qui ont trouvé qu'un dosage de $0.3 \%$ de PC réduit la contrainte tangentielle de la pâte de $70 \%$ suivi par une diminution de la viscosité de la pâte.

D'autre part la valeur de la viscosité d'un ciment plus fin est supérieure à celle d'un ciment à faible finesse. Cet effet sur les particules fines a également été étudié par Nawa et al. [18] où la viscosité des pâtes de ciment accrue avec la fraction fine ( $\leq 10 \mathrm{um}$ ) de ciment. Cela est dû à l'augmentation du montant de superplastifiant adsorbé lorsque la finesse du ciment augmente.

\subsection{Résistance mécanique}

De la "figure 3", On observe que la valeur de la résistance à la compression du mortier à base de ciment fin est beaucoup plus élevée que celle d'un mortier à base de ciment à gros grains car les ciments fins s'hydratent rapidement ce qui accélère la formation des hydrates qui offrent à la matrice cimentaire une meilleure résistance mécanique à la compression. Ainsi pour des finesses élevées, le clincker adhère mieux au calcaire [5].

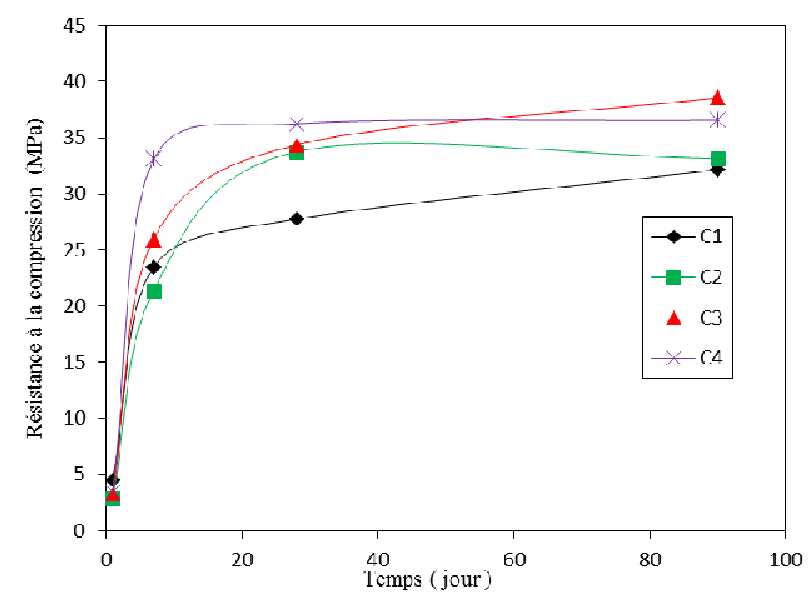

Fig.3. Effet de la finesse sur la résistance mécanique des mortiers non adjuvantés.

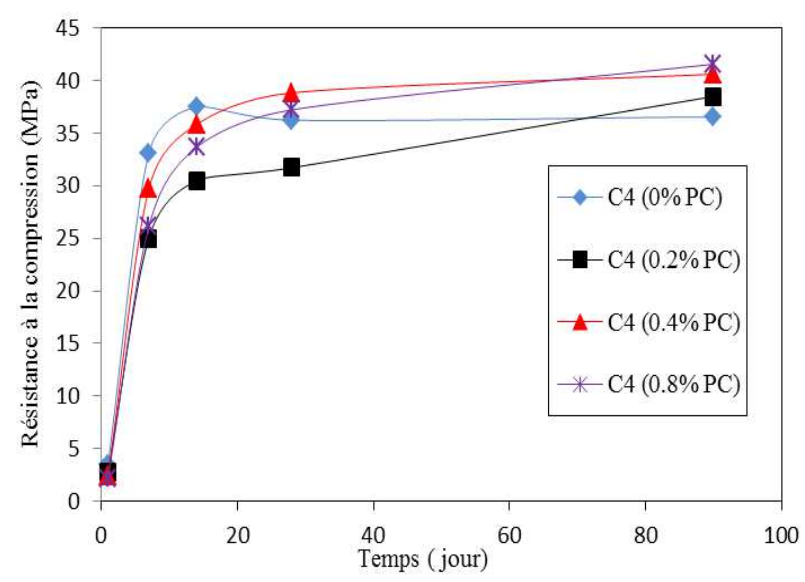

Fig.4. Effet du dosage en polycarboxylate sur la résistance mécanique des mortiers à base de ciment $\mathrm{C} 4$.
D'après les résultats illustrés sur la "figure 4", on peut constater qu'au jeune âge la valeur de la résistance du mortier non adjuvanté est presque similaire à celles des mortiers adjuvantés, mais après 28 jours l'effet du superplastifiant améliore les propriétés mécaniques du mortier en enregistrant des résistances mécaniques importantes surtout pour les dosages $0.4 \%$ et $0.8 \%$ PC.

\section{Conclusion}

D'après les résultats obtenus nous pouvons constater que l'effet de la finesse du ciment utilisé est très signifiant en présence de l'addition minérale calcaire et du superplastifiant Polycarboxylate Où :

- Le seuil de cisaillement et la viscosité du mortier augmentent lorsque la finesse du ciment passe de $3026 \mathrm{~cm}^{2} / \mathrm{g}$ à $4000 \mathrm{~cm}^{2} / \mathrm{g}$ et pour des dosages en Polycarboxylate inférieurs à $0.8 \%$.

- L'augmentation de la finesse améliore la résistance mécanique des mortiers testés .

- L'ajout du Polycarboxylate a un effet bénéfique sur le développement des résistances mécaniques surtout pour des dosages inférieurs ou égaux à $0.8 \%$.

\section{References}

1. Yahia A., Tanimura M., Shimoyama Y., Réological properties of highly flowable mortar containing limestone filler-effect of powder content and $w / c$ ratio cement and concrete research, 35, 532-539,(2005).

2. Bonavetti V., Donza H., Rahhal V., Irassar E., Influence of initial curing on the properties of concrete containing limestone blended cement ,Cement and Concrete Research , 30, 703-708, (2000).

3. Menendez G., Bonavetti V., Irassar EF., Strength development of ternary blended cement with limestone filler and blast-furnace slag, Cement and Concrete Composition, 25, 61-67, ( 2001).

4. Zhang X., Han J., The effect of ultra-fine admixture on the rheological property of cement paste, Cement and Concrete Research, 30, 827-30, (2000).

5. Tsivilis S., Chaniotakis E., Badogiannis E., Pahoulasa G., Ilias A. , A study on the parameters affecting the properties of Portland limestone cements, Cement and Concrete Composites, 21, 107-116,(1999).

6. Lin F., Meyer C., Hydration kinetics modeling of Portland cement considering the effects of curing temperature and applied pressure, Cement and Concrete Research , 39, 255-265,(2009).

7. Soroka I., Stern N., Calcareous fillers and the compressive strength of Portland cement, cement and concrete research, 6, 367-376,(1976).

8. Lothenbach B., Le Saout G., Gallucci E., Scrivener $\mathrm{K}$., Influence of limestone on the hydration of Portland cements, Cement and Concrete Research, 38, 848-860,(2008).

9. Mounanga P. , Irfan M. , Khokhar A. , Elhachem Rana loukili A., Improvement of the early-age reactivity of fly ash and blast furnace slag 
cementations systems using limestone filler, Materials and structures , 44, 437 - 453 ,(2011).

10. Tsivilis S., Chaniotakis E., Kakali G., Batis G., An analysis of the properties of Portland limestone cements and concrete, Cement and Concrete Composites, 24, 371-378,(2002).

11. Heikel M., El-didamony H., Morsy M.S., limestone filled pozzolanic cement, Cement and Concrete Research, 30,1827-1834, (2000).

12. Puertas F., Santos H., Palacios M., MartınezRamirez S., Polycarboxylate superplasticiser admixtures: effect on hydration, microstructure and rheological behaviour in cement pastes, Advances in Cement Research, 17, No. 2, 77-89, (2005).

13. Bjornstrom J., Chandra S., Effect of superplasticizers on the rheological properties of cements, Materials and Structures / Matériaux et Constructions,36, 685-692, (2003).

14. Aiad I., Influence of time addition of superplasticizers on the rheological properties of fresh cement pastes, Cement and Concrete Research, 33, 1229 - 1234, (2003).

15. Golaszewki JG., Szwabowski J., Influence of superplasticizers on rheological behaviour of fresh cement mortars, Cement and Concrete Research, 34, 235-248,(2004).

16. Heikal M., Morsy M.S., Aiad I, Effect of treatment temperature on the early hydration characteristics of superplasticized silica fume blended cement pastes, Cement and Concrete Research ,35, 680-687, (2005).

17. Courrazé G., Grossiord JL., Initiation à la rhéologie, Tec\& Doc, (2000).

18. Nawa T., Eguchi H., Effect of cement characteristics on the fluidity of cement paste containing an organic admixture, Proceedings of the 9th International Congress on the Chemistry of Cements, 4, 597-603, (1992). 